\title{
REVIEW ON FACE DETECTION AND RECOGNITION TECHNOLOGY BASED ON NEURAL NETWORK
}

\author{
Abhishek Yadav ${ }^{1 *}$, Sachin Mahajan² \\ ${ }^{*}$ P. G Scholar, Department of Computer Science and Engineering,Jawaharlal institute of technology Borawan, \\ Khargone ( $m p)$, India \\ ${ }^{2}$ Professor, Department of Computer Science and Engineering, Jawaharlal institute of technology Borawan, Khargone \\ ( $m p)$, India \\ *1Email: abhisheky13@gmail.com ; ${ }^{2}$ sachinmahajan248@gmail.com
}

*Corresponding Author: -

Email ID - abhisheky13@gmail.com

\begin{abstract}
: -
In this paper, feature extraction and facial recognition are studied in order to resolve problems like highdimension problem, small size samples and no-linear separable problem that exist in facial recognition technology. In the part of feature extraction we use a HGPP algorithm, to extract the input features in building a face recognition system. The neural network, which represents brilliant performance on small training sets, non-linear separable and high-dimension pattern recognition problems in the recognition stage, is used for pattern classification. The proposed approach is validated with the ORL database. Experimental results demonstrate the effectiveness of this method in the performance of face recognition.
\end{abstract}

Keywords: - Face Detection, Recognition, Neural Network

\section{(c) $(\$)$}




\section{INTRODUCTION:}

Nowadays, Biometric Recognition, or simply Biometrics, which refers to automatic recognition of individuals based on their physiological and/or behavioral characteristics, is a prominent field of research. Among all the biometrics like: face, fingerprint, hand geometry, iris, signature, DNA etc. face has an outstanding importance. Especially because of its contactless property, applications using this biometric are widely useful in surveillance cameras in public places like airports, banks, train stations, etc. Cameras providing inputs for such a system in public places are usually working constantly. Continues recordings from these cameras produce huge amount of video data. Considering a person passing by such a surveillance camera, a sequence of images of that person is captured by the camera. Faces in such videos can be detected in real-time but using all of the detected faces in almost any computer vision application is extremely demanding. Many of the detected faces are useless due to problems like not facing the camera, blurriness of the face, darkness of the face, small size of the face, and changes in the status of facial components (like closeness of the eyes, openness of the mouth). Using such face images would produce erroneous results in almost any facial analysis system. Furthermore, there are many faces that resemble each other very closely and keeping only some of them may suffice. Therefore, it is reasonable and necessary to use a mechanism for assessing the quality of the face images, i.e. face quality assessment [1]. This mechanism should discard useless face images and summarize the input video sequence to smaller sets containing some of the most expressive images of the video. These summarized sets, containing the most expressive images, are denoted Face-Log(s) [2].

Face recognition systems are part of facial image processing applications and their significance as a research area is increasing recently. They use biometric information of the humans and are applicable easily instead of fingerprint, iris, signature etc., because these types of biometrics are not much suitable for any collaborative people. Face recognition systems are usually applied and preferred for people and security cameras in metropolitan life. These systems can be used for crime prevention, video surveillance, person verification, and similar security activities.

Face recognition system is a complex image-processing problem in real world applications with complex effects of illumination, occlusion, and imaging condition on the live images. It is a combination of face detection and recognition techniques in image analyzes. Detection application is used to find position of the faces in a given image. Recognition algorithm is used to classify given images with known structured properties, which are used commonly in most of the computer vision applications. These images have some known properties like; same resolution, including same facial feature components, and similar eye alignment. These images will be referred as "standard image" in the further sections. Recognition applications uses standard images and detection algorithms detect the faces and extract face images which include eyes, eyebrows, nose, and mouth. That makes the algorithm more complicated than single detection or recognition algorithm. The first step for face recognition system is to acquire an image from a camera. Second step is face detection from the acquired image.

As a third step, face recognition that takes the face images from output of detection part. Final step is person identity as a result of recognition part [3]. An illustration of the steps for the face recognition system is given in Figure 1.1

Acquiring images to computer from camera and computational medium (environment) via frame grabber is the first step in face recognition system applications. The input image, in the form of digital data, is sent to face detection algorithm part of software for extracting each face in the image. Many methods are available for detecting faces in the images in the literature [1-10]. The available methods could be classified into two main groups as; knowledge-based and appearancebased methods. Briefly, knowledge-based methods are derived from human knowledge for features that makes a face.

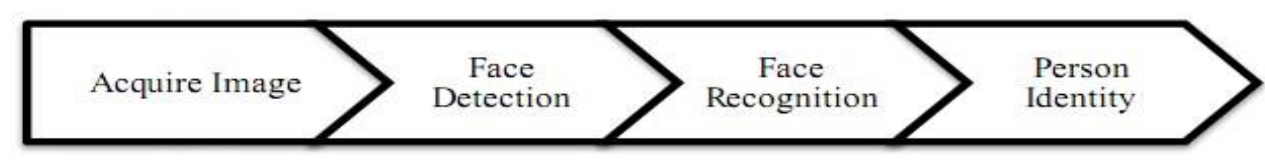

Fig. 1.1 Steps of Face Recognition System Applications

Methods for face detection and recognition systems can be affected by pose, presence or absence of structural components, facial expression, occlusion, image orientation, imaging conditions, and time delay (for recognition). Available applications developed by researchers can usually handle one or two effects only; therefore they have limited capabilities with focus on some well-structured application. A robust face recognition system is difficult to develop which works under all conditions with a wide scope of effect.

The face is our primary focus of attention in social intercourse, playing a major role in conveying identity and emotion. Although the ability to infer intelligence or character from facial appearance is suspect, the human ability to recognize faces is remarkable. We can recognize thousands of faces learned throughout our lifetime and identify familiar faces at a glance even after years of separation.

This skill is quite robust, despite large changes in the visual stimulus due to viewing conditions, expression, aging, and distractions such as glasses, beards or changes in hair style.

Face recognition has become an important issue in many applications such as security systems, credit card verification and criminal identification. For example, the ability to model a particular face and distinguish it from a large number of stored face models would make it possible to vastly improve criminal identification. Even the ability to merely detect faces, as opposed to recognizing them, can be important. Detecting faces in photographs for automating color film development can be very useful, since the effect of many enhancement and noise reduction techniques depends on the image content[4]. 


\subsection{Task for Face recognition:}

(a) Verification (one-to-one matching): When presented with a face image of an unknown individual along with a claim of identity, ascertaining whether the individual is who he/she claims to be.

(b) Identification (one-to-many matching): Given an image of an unknown individual, determining that person's identity by comparing (possibly after encoding) that image with a database of (possibly encoded) images of known individuals.

There are numerous application areas in which face recognition can be exploited for these two purposes, a few of which are outlined below.

(a) Security (access control to buildings, airports/seaports,ATM machines and border checkpoints [2]; computer/ network security; email authentication on multimedia workstations).

(b) Surveillance (a large number of CCTVs can be monitored to look for known criminals, drug offenders, etc. and authorities can be notified when one is located; for example, this procedure was used at the Super Bowl 2001 game at Tampa, Florida [3]; in another instance, according to a CNN report, two cameras linked to state and national databases of sex offenders, missing children and alleged abductors have been installed recently at Royal Palm Middle School in Phoenix, Arizona [4]).

(c) General identity verification (electoral registration, banking, electronic commerce, identifying new borne, national IDs, passports, drivers' licenses, employee IDs).

(d) Criminal justice systems (mug-shot/booking systems, post-event analysis, forensics).

(e) Image database investigations (searching image databases of licensed drivers, benefit recipients, missing children, immigrants and police bookings).

(f) "Smart Card" applications (in lieu of maintaining a database of facial images, the face-print can be stored in a smart card, bar code or magnetic stripe, authentication of which is performed by matching the live image and the stored template) [5].

(g) Multi-media environments with adaptive human computer interfaces (part of ubiquitous or context aware systems, behavior monitoring at childcare or old people's centers, recognizing a customer and assessing his needs).

(h) Video indexing (labeling faces in video) [6,7].

(i) Witnesses face reconstruction [8].

\subsubsection{Steps of Face Recognition}

In identification problems, the input to the system is an unknown face, and the system reports back the determined identity from a database of known individuals, whereas in verification problems, the system needs to confirm or reject the claimed identity of the input face. Face perception is an important part of the capability of human perception system and is a routine task for humans, while building a similar computer system is still an on-going research area. For example, recognition of face images acquired in an outdoor environment with changes in illumination and/or pose remains a largely unsolved problem. In other words, current systems are still far away from the capability of the human perception system.

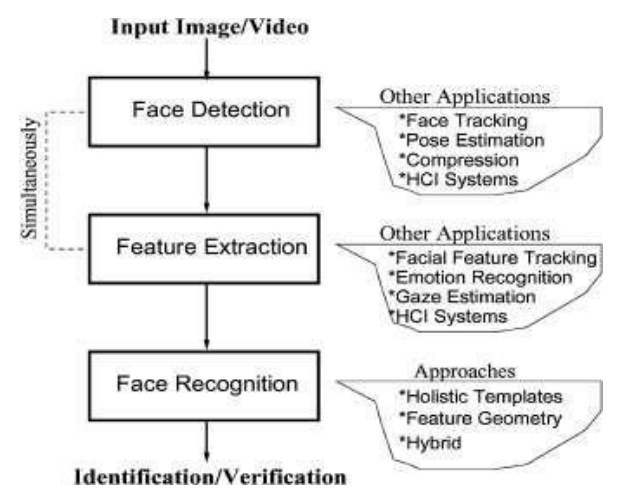

As illustrated in Figure 3.3, the problem of automatic face recognition involves three key steps/subtasks:

(a) Detection and rough normalization of faces,

(b) Feature extraction and accurate normalization of faces,

(c) Identification and/or verification.

Once the training is over, the network is ready to recognize face presented at its input. For recognizingthe face two options are provided. If user wants to recognize the existing image,then it can be loaded from memory. As the user selects the image, the face recognition method works andreturns the face part of the image. And other option is to capture the live image. Image is captured from the web cam. Once asked to recognize, it captures theimage and finds the face part in it. Then the edge detection, thinning, and token generation are performed.Then it classifies the given tokens into one of three faces it learned during training. It gives percentageof recognition to each face with highest percentage closely matching and lowest to the farthest matchingand the closest match is considered as the result. The recognition process is implemented as per the outlinegiven in the flow chart in Fig.3.9. 


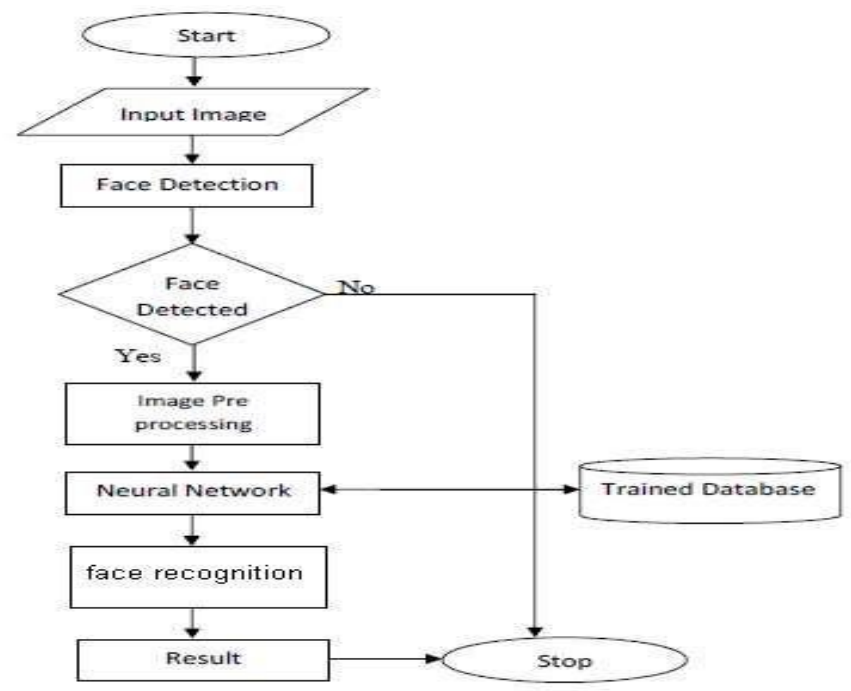

Fig. 3.9 Flow chart of face Recognition System

Above we show the flow chart of face recognition system. In this chart we firstly give input image then face deducted. When faces are detected the process will continue otherwise its stop. If face are detected then image are pre processing and go to neural network there image is trained from database and face recognized and we have final result of matched face.

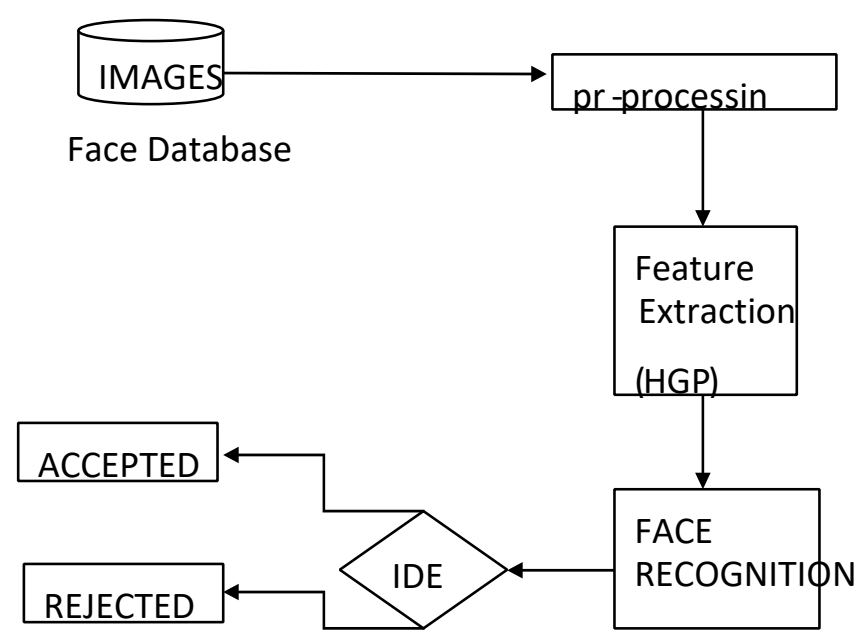

\section{RESULT ANALYSIS}

\subsection{Result Analysis}

There is we create the accuracy graph of face recognition. There we have insert images and that image will be recognize through the database and then we create graph of accuracy and there is proposed algorithm match face with purity and robust matching of faces and that will be robust face recognition.

\subsection{Data set:}

We have used ORL data set containing 400 images. The experimental results have shown that out of these 400 images, a total of 388 images have been identified correctly and the remaining two have been identified incorrectly.

\subsection{Accuracy:}

Therefore the accuracy of the proposed method is Accuracy $=97 \%$

\section{Conclusion}

The experimental results showed that the method in this paper worked well on ORL face database, and also had a good anti-noise performance, although at high intensity noise, it also needed to maintain a high recognition rate. So, this method has good capability of resolving high-dimension problems, small size samples problems and no-linear separable problem. The feature vectors are only extracted from gray-scale, so a real-time recognition system needs to be researched continuously.

\section{REFERENCES}

[1].N. Revathy, 2T.Guhan, 2012, "FACE RECOGNITION SYSTEM USING BACK PROPAGATION ARTIFICIAL NEURAL NETWORKS”, Proc. International Journal of Advanced Engineering Technology, pp.321-324, India. 
[2].K. Seo, W. Kim, C. Oh and J. Lee, 2002, "Face Detection And Facial Feature Extraction Using Color Snake", Proc. ISIE 2002 - 2002 IEEE International Symposium on Industrial Electronics, pp.457-462, L 'Aquila, Italy.

[3].C. Lin, 2005, "Face Detection By Color And Multilayer Feedforward Neural Network", Proc. 2005 IEEE International Conference on Information Acquisition, pp.518-523, Hong Kong and Macau, China.

[4].M. A. Berbar, H. M. Kelash and A. A. Kandeel, 2006, "Faces And Facial Features Detection In Color Images", Proc. Geometric Modeling and Imaging - New Trends (GMAI'06), pp.209-214, London, UK.

[5].S. Kherchaoui and A. Houacine, 2010, "Face Detection Based On A Model Of The Skin Color With Constraints And Template Matching”, Proc. 2010 International Conference on Machine and Web Intelligence, pp. 469 - 472, Algiers, Algeria.

[6].Chandrappa D N, M Ravishankar, D R RameshBabu, 2011, "Face Detection in Color Images using Skin Color Model Algorithm based on Skin Color Information”, 2011 3rd International Conference on Electronics Computer Technology (ICECT 2011),pp. $254-258$, India.

[7].C. Aiping, P. Lian, T. Yaobin and N. Ning, 2010, "Face Detection Technology Based On Skin Color Segmentation And Template Matching", Proc. 2010 Second International Workshop on Education Technology and Computer Science, pp.708-711, Wuhan, China.

[8].Ali Atharifard, and SedighehGhofrani, 2011, "Robust Component-based Face Detection Using Color Feature", Proc. Proceedings of the World Congress on Engineering 2011 Vol II, WCE 2011, July 6 - 8, 2011, London, U.K.

[9].Yi Zhu and Florin Cutu, 2009, "Face Detection using Half-Face Templates", Proc. The Ninth International Conference on Electronic Measurement \& Instruments ICEMI, Indiana University, USA. 